\title{
Spacecraft Alignment Determination and Control for Dual Spacecraft Precision Formation Flying
}

\author{
Philip Calhoun, Anne-Marie Novo-Gradac, Neerav Shah
}

NASA Goddard Space Flight Center 


\section{Overview}

- VT Concept

- Astrometric Alignment Concept for Virtual Telescopes (VT)

- Proposed Missions (MASSIM, New Worlds Observer)

- Stability Requirements and Measurement Models

- Attitude and Translation Stability Requirements

- Optical Alignment and Ranging System Measurement Models

- Dynamics and Controls Framework for GN\&C Design

- Dynamics Model Formulation

- Inertial Measurement Models (IRU, Accelerometers)

- Case Study: GN\&C Design for a Heliophysics Mission

- Navigation Modes for Fine Alignment Acquisition

- GN\&C Architecture Comparison

- Conclusions 


\section{VT Concept}

- Formation flying missions seek to advance science imaging by utilizing precision dual spacecraft formation flying. ( "Virtual" Telescope (VT))

- Milli-Arc-Second Structure Imager (MASSIM) (Astrophysics X-ray imaging) (Sep 1000 km)

- New Worlds Observer (NWO) (exoplanet mission) (Sep 25,000 km)

- Heliophysics concept missions for Solar Coronagraphs and Solar imaging (Sep. 50m - 500m)

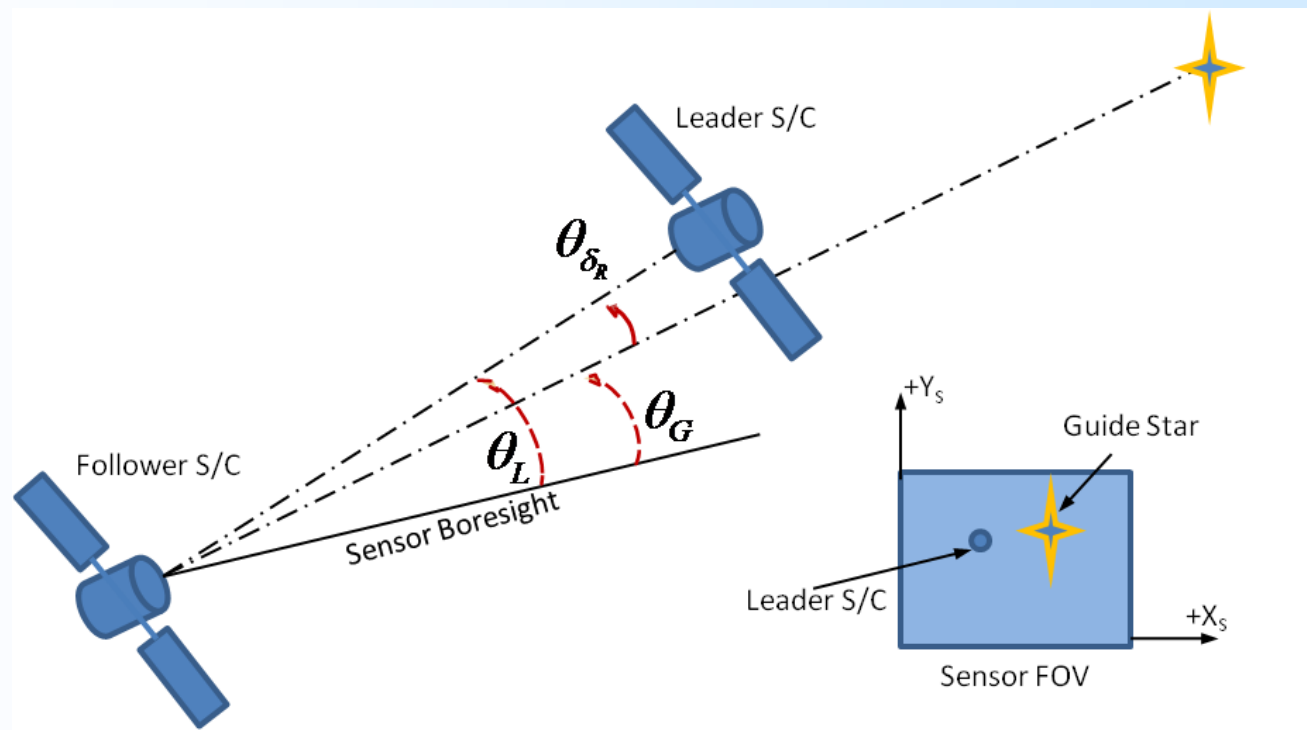

Dual Spacecraft Precision Astrometric Alignment Sensing Architecture

Objective: Develop models for a complete GN\&C design framework of VT architectures 


\section{VT Attitude and Translation Stability Requirements}

- Science detector image smear and depth of field stability model

- Considers detector and optics not co-located with S/C mass center

$$
\begin{gathered}
\vec{\delta}_{I}=\left[\begin{array}{c}
s_{x} \\
s_{y} \\
d
\end{array}\right]=\left[\vec{P}_{D}+\vec{P}_{D O}+\vec{\delta}_{R}-\vec{P}_{O}+R\left(\vec{\theta}_{O}\right) \vec{P}_{O}+f\left(R\left(\vec{\theta}_{O}\right)\right) \vec{P}_{O D}\right]-R\left(\vec{\theta}_{D}\right) \vec{P}_{D} \\
f\left(R\left(\vec{\theta}_{O}\right)\right)=\left[I+{ }^{n} \tilde{\theta}_{O}\right], \quad{ }^{n} \vec{\theta}_{O}=\Phi \vec{\theta}_{O}, \quad \Phi \text { is diag matrix, } 0<\phi_{i}<1
\end{gathered}
$$

$\vec{\delta}_{I}=\left[\begin{array}{l}s_{x} \\ s_{y} \\ d\end{array}\right]=\widetilde{P}_{D}^{x} \vec{\theta}_{D}+\left[\Phi \tilde{P}_{D O}^{x}-\widetilde{P}_{O}^{x}\right] \vec{\theta}_{O}+\vec{\delta}_{R}$

$\vec{\delta}_{L}=\left[\begin{array}{c}l_{x} \\ l_{y} \\ r_{l}\end{array}\right]=\tilde{P}_{L}^{x} \vec{\theta}_{D}+\left[\Phi \tilde{P}_{L B}^{x}-\tilde{P}_{B}^{x}\right] \vec{\theta}_{O}+\vec{\delta}_{R}$

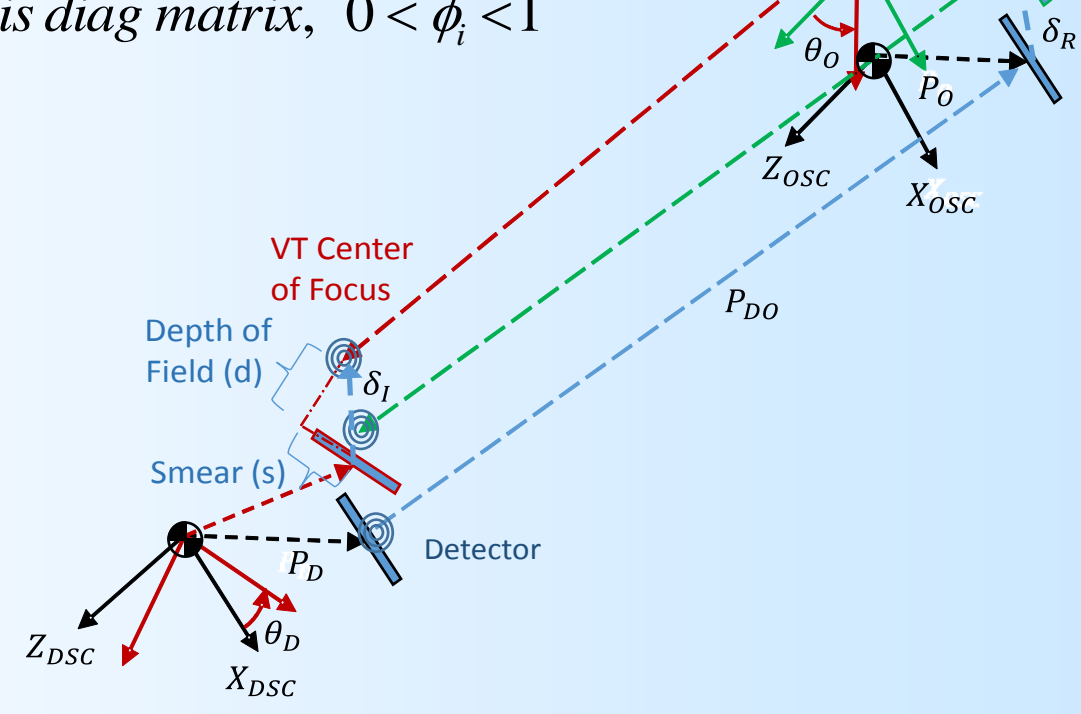

- Same model is used for laser centration and ranging measurements 


\section{Alignment Camera Measurement Model}

- Measurement model for Alignment Camera (AC) to track laser beacon bearing angles.

- AC used for acquisition of Laser Centration and Ranging elements.

$$
\vec{P}_{C B}=\left[\vec{P}_{C}+\vec{P}_{C O}^{\prime}+\vec{\delta}_{R}+R\left(\vec{\theta}_{O}\right) \vec{P}_{B}\right]-R\left(\vec{\theta}_{D}\right) \vec{P}_{C}
$$

Laser beacon centroids on the camera image

$$
\left[\theta_{x}, \theta_{y}\right]=\operatorname{atan}\left(\frac{\widehat{P}_{C B}(2)}{\widehat{P}_{C B}(3)}\right), \operatorname{atan}\left(\frac{\widehat{P}_{C B}(1)}{\widehat{P}_{C B}(3)}\right)
$$

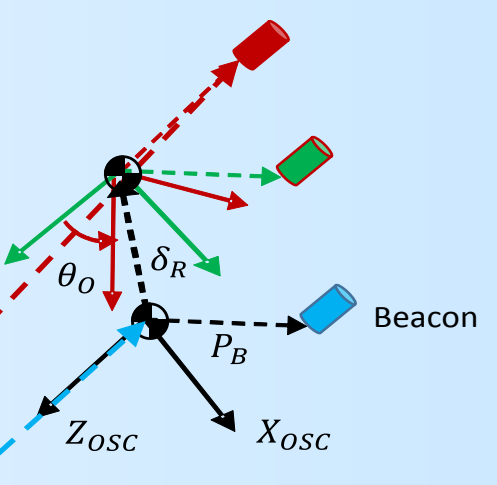




\section{VT Dynamics Framework for GN\&C Design}

- Dual Spacecraft Relative Dynamics (Based on Luquette's work)

- Restricted Three-body Framework

- Mods: Additional gravitational bodies, and express equations in terms of Follower

differential acceleration, expressed in an inertial frame,

$$
\begin{aligned}
& \ddot{\bar{x}}=\ddot{\bar{r}}_{F}-\ddot{\bar{r}}_{L} \\
& \ddot{\bar{r}}_{F}=-\sum_{i=1}^{n} \mu_{i} \frac{\bar{r}_{i F}}{\left\|\bar{i}_{i F}\right\|^{3}}+\bar{f}_{\text {solar }_{,} F}+\bar{f}_{\text {pert }, F}+\bar{u}_{\text {thrust }, F} \\
& \ddot{\bar{r}_{L}}=-\sum_{i=1}^{n} \mu_{i} \frac{\bar{r}_{i L}}{\left\|\bar{r}_{i L}\right\|^{3}}+\bar{f}_{\text {solar }, L}+\bar{f}_{\text {pert }, L}+\bar{u}_{\text {thrust }, L}
\end{aligned}
$$

Can be simplified in terms of follower S/C, following derivation by Luquette.

- Assume $\|\bar{x}\| \ll\left\|\bar{r}_{i F}\right\|, \quad \bar{x}=\bar{R}^{\text {ref }}+\bar{\delta}_{R} \quad$ and remove higher order terms.

$$
\begin{aligned}
& \ddot{\bar{\delta}}_{R}=\Gamma_{G G} \bar{\delta}_{R}+\Gamma_{G G} \bar{R}^{r e f}+\bar{u}_{R} \\
& \Gamma_{G G}=-\sum_{i=1}^{n} \frac{\mu_{i}}{\left\|\bar{r}_{i F} r e f\right\|^{3}}\left([I]-3 \hat{r}_{i F} \text { ref }\left[\hat{r}_{i F} r e f\right]^{T}\right)
\end{aligned}
$$




\section{VT Dynamics Framework for GN\&C Design}

- Inertial Measurement Sensor (Accelerometers)

The acceleration, $\ddot{\bar{\delta}}_{F} \mathrm{~m}$, at a specific sensor location, $\overline{\mathrm{r}}_{\mathrm{A}}$, can be represented as, $\ddot{\bar{\delta}}_{F} \mathrm{~m}=\ddot{\bar{\delta}}_{F}+\bar{\omega}_{\mathrm{F}} \times\left(\bar{\omega}_{\mathrm{F}} \times \overline{\mathrm{r}}_{\mathrm{A}}\right)+\bar{\omega}_{\mathrm{F}} \times \overline{\mathrm{r}}_{\mathrm{A}}+\bar{b}_{A}+\bar{v}_{A}$

Acceleration can be expressed in terms of forces / torques on S/C $\ddot{\delta_{F}}=\overline{\mathrm{u}}_{\mathrm{F}_{\mathrm{T}_{0}}}+{\overline{\delta \mathrm{u}_{\mathrm{T}}}}_{\mathrm{F}_{\mathrm{T}}}+\overline{\mathrm{u}}_{\mathrm{F}_{\mathrm{E}}} \quad \dot{\bar{\omega}}_{\mathrm{F}}=\mathrm{I}_{\mathrm{F}}^{-1}\left(\overline{\mathrm{T}}_{\mathrm{T}_{0}}+\overline{\delta \mathrm{T}}_{\mathrm{F}_{\mathrm{T}}}+\overline{\mathrm{T}}_{\mathrm{F}_{\mathrm{E}}}\right)$

And reduced to following linear form,

$$
\begin{aligned}
\ddot{\bar{\delta}}_{F}^{\mathrm{m}} & =\left([I]-r_{A}^{x} \mathrm{I}_{\mathrm{F}}^{-1} r_{T}^{x} \mathrm{~m}_{\mathrm{F}}\right) \overline{\mathrm{u}}_{\mathrm{F}_{\mathrm{T}}}+\left([I]-r_{A}^{x} \mathrm{I}_{\mathrm{F}}^{-1} r_{T}^{x} \mathrm{~m}_{\mathrm{F}}\right){\overline{\delta \mathrm{u}_{\mathrm{T}}}}_{\mathrm{T}_{\mathrm{T}}} \\
& +\left([I]-r_{A}^{x} \mathrm{I}_{\mathrm{F}}^{-1} r_{E}^{x} \mathrm{~m}_{\mathrm{F}}\right) \overline{\mathrm{u}}_{\mathrm{F}_{\mathrm{E}}}+\bar{b}_{A}+\bar{v}_{A}
\end{aligned}
$$

- Inertial Reference Unit (Accelerometers)

$$
\dot{\bar{\theta}}=\bar{\omega}_{\mathrm{F}}^{\mathrm{m}}-\bar{b}_{\omega_{F}}+\bar{v}_{\omega_{F}}
$$




\section{Case Study: GN\&C Design for Heliophysics Mission}

\section{Closed Loop GN\&C Simulation Case Study - Photon Sieve}

- GN\&C design framework applied to an example problem to illustrate trades inherent in PFF for VT.

- Photon Sieve Optics (difractive optics, $\sim 0.5 \mathrm{~m}$ aperture). Solar Imaging at milli-arc-sec level

Table 1, Photon Sieve VT Alignment Requirements and Component Specifications

\begin{tabular}{|c|c|c|c|}
\hline Parameter & Requirement (3s) & Component & Specification (3s) \\
\hline Image Smear & 6 microns & Laser Centration & 30 microns \\
\hline Depth of Field & $1 \mathrm{~mm}$ & Laser Ranging & $0.5 \mathrm{~cm}$ \\
\hline S/C separation & $200 \mathrm{~m}$ & Microthruster & $5 \mathrm{mN}$-sec (min Impulse) \\
\hline $\begin{array}{l}\text { Pointing Stability } \\
\text { (Optics S/C) }\end{array}$ & $\begin{array}{l}5 \text { milli-arc sec (Sun) } \\
10 \text { arc-sec (roll) }\end{array}$ & Fine Sun Sensor & 30 milli arc-sec \\
\hline $\begin{array}{l}\text { Pointing Stability } \\
\text { (Detector S/C) }\end{array}$ & 10 arc-sec & Star Tracker & $\begin{array}{l}6 \text { arc-sec (transverse) } \\
30 \text { arc-sec (boresight) }\end{array}$ \\
\hline
\end{tabular}

- State estimation: Extended Kalman Filter, continuous state propagation, discrete measurements

- Sequential Measurement updates to avoid numerical issues of large matrix inverses

- Separate PID Control for each of 9 DoF, (3) Relative translation, (3) Optics S/C Att, (3) Optics S/C Att

- All Measurement and Actuator models include random + systematic ( $\left(^{\text {st }}\right.$ order Markov) errors 


\section{Case Study: GN\&C Design for Heliophysics Mission}

- Simulation of Navigation modes (Leader/Follower) illustrates fine align acquisition

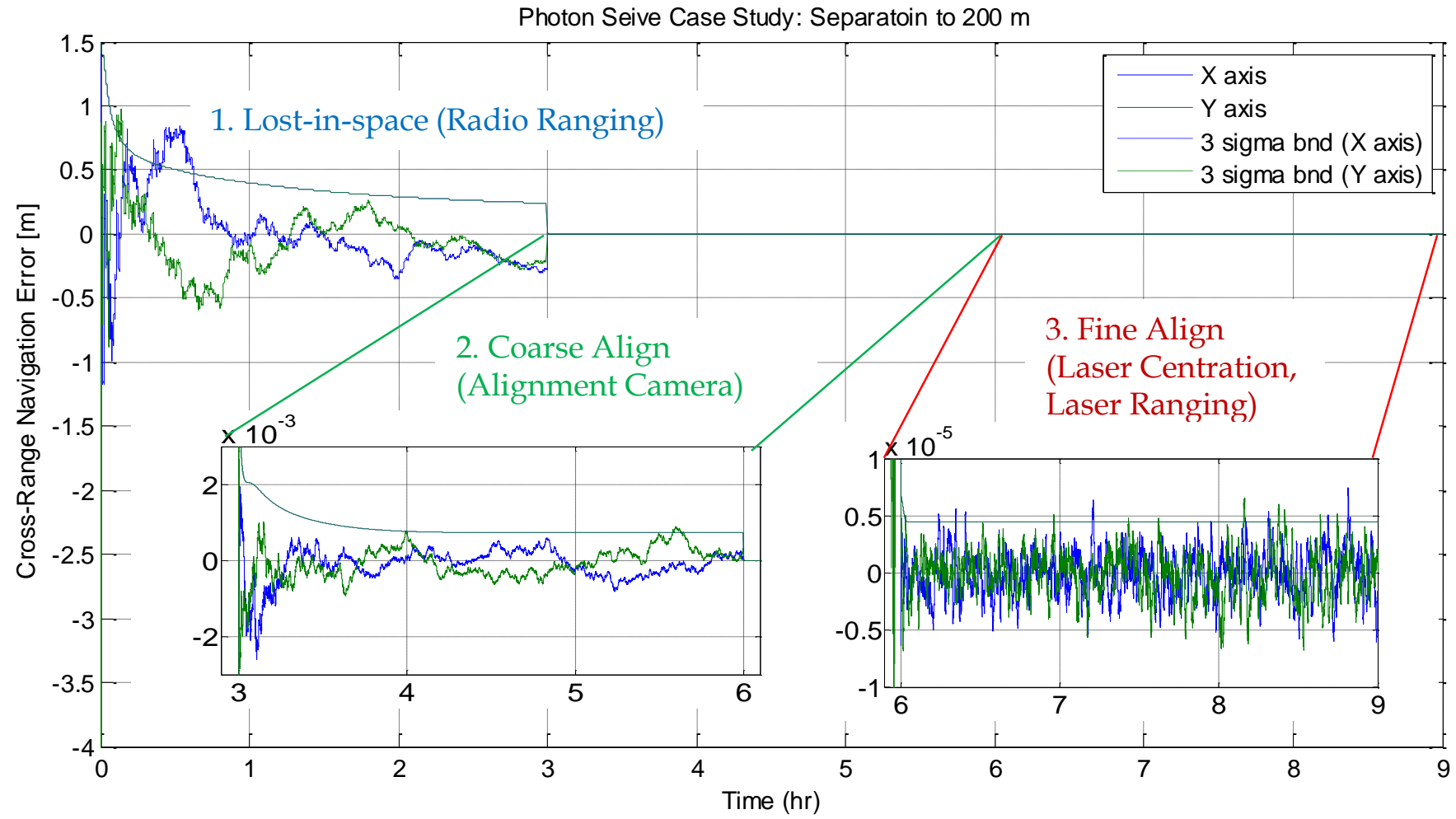

1. Lost-in-space: Radio Range $(60 \mathrm{~cm})$, Radio bearing $(9 \mathrm{deg})$

2. Coarse Align: Alignment Camera (50 arc-sec), Star Tracker (ST) $(6,6,30$ arc-sec)

3. Fine Align: Laser Centration $(30 \mu \mathrm{m})$, Laser Ranging: $(1 \mathrm{~cm}), \mathrm{ST}(6,6,30$ arc-sec), Sun Sen $(10 \mathrm{e}-3$ arc-sec) 


\section{Case Study: GN\&C Design for Heliophysics Mission}

- Evaluation of Leader/Follower and Partition Architecture illustrates GN\&C trades

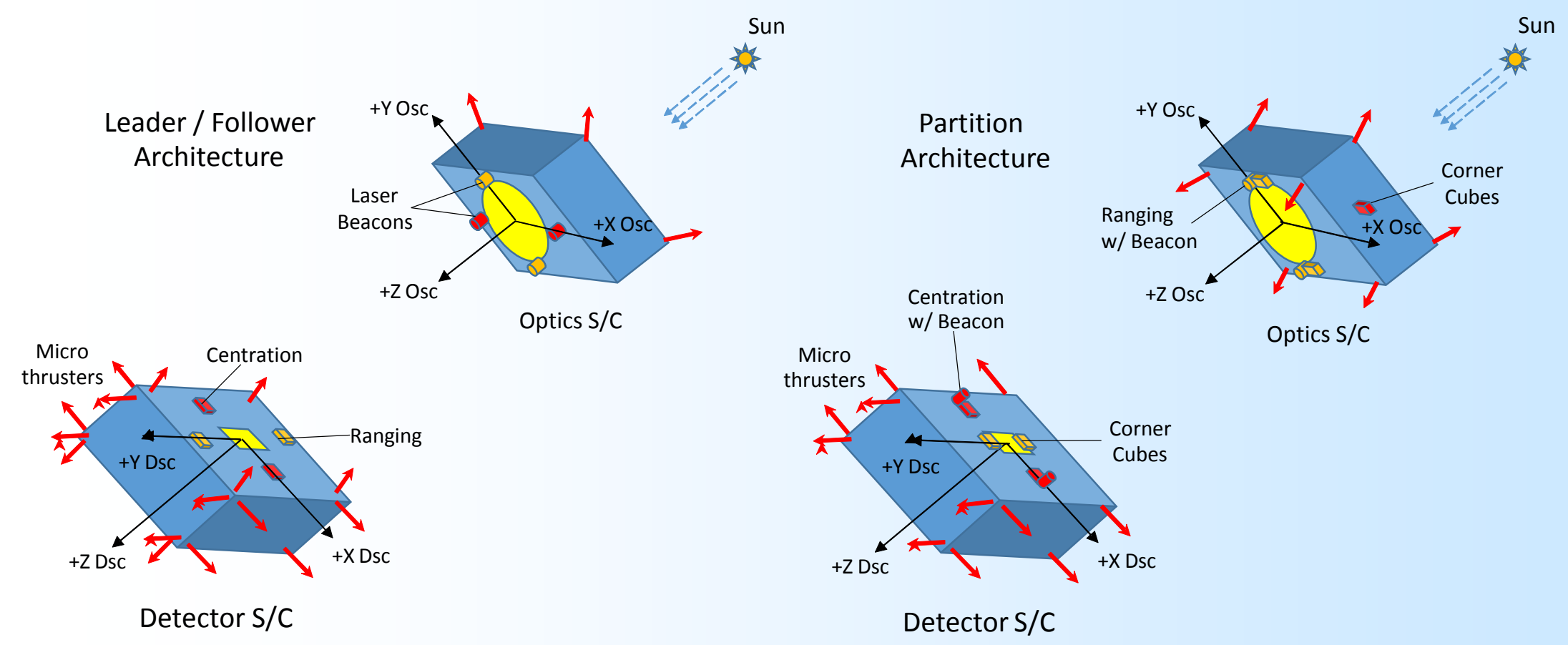

Leader/Follower architecture has two possible deficiencies

- Comm link required to send Optics S/C Attitude to EKF on Detector S/C. Comm delay/timing sync

- Requires Full 6 DoF control on Detector S/C. Thruster coupling may result in poor performance

Partition architecture: Control/ Estimation is partitioned among the two S/C

- Avoids multi-platform attitude coupling in the measurement process 


\section{Case Study: GN\&C Design for Heliophysics Mission}

- Evaluation of Leader/Follower and Partition Architecture illustrates GN\&C trades
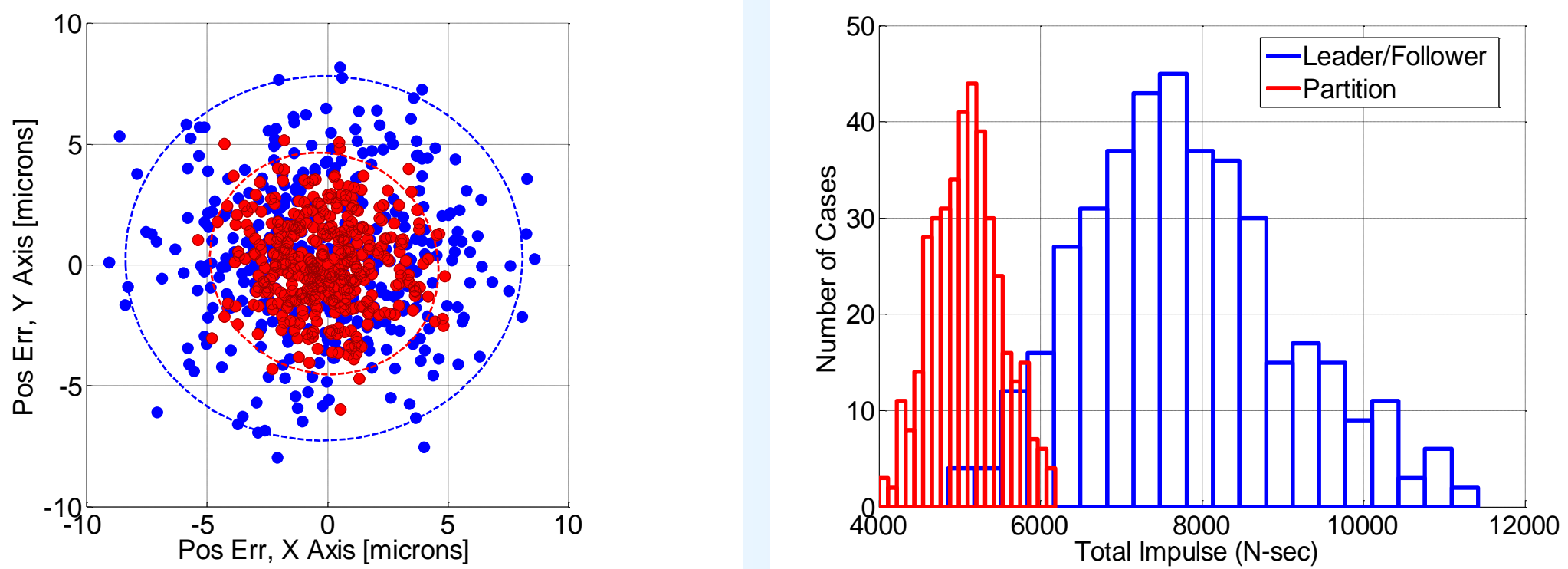

Figure 5 - Performance results of two representative GN\&C architectures for the VT

- Decoupling of laser centration/ranging measurements from the $\mathrm{S} / \mathrm{C}$ attitude, ( sensor positioning )

- Improved transverse alignment observability / performance in the Partition architecture.

- Partition architecture performance meets Photon Sieve alignment requirements

- $\sim 5 x$ error reduction obtained from model-based estimation over laser centration measurements.

- Total impulse for PFF (5 year) is reduced 35\%. Solar pres along VT axis (Optics $\mathrm{S} / \mathrm{C}$ is $1 / 2$ mass of Detector $\mathrm{S} / \mathrm{C}$ ) 


\section{Conclusions}

- Developed General 9 DoF GN\&C framework dual S/C PFF for application to VT missions

- GN\&C performance assessment for a representative Heliophysics VT imaging mission concept illustrates the potential trade-offs inherent in the choice of system architecture for GN\&C design and mission concept.

Acknowledgments

- The authors would like to acknowledge Joe Davila for his support, technical oversight, and assistance in understanding the scientific objectives of the Photon Sieve concept, and Michael Johnson for his support of the IRAD effort to accomplish this work. 